Few-Body Systems 0, 1-3 (2007)

FewBody

Systems

(C) by Springer-Verlag 2007 Printed in Austria

\title{
Spin dependent parton distributions and structure functions
}

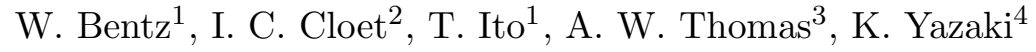 \\ ${ }^{1}$ Department of Physics, School of Science, Tokai University, Hiratsuka-shi, Kanagawa 259- \\ 1292, Japan \\ ${ }^{2}$ Physics Division, Argonne National Laboratories, Argonne, IL 60439-4843, USA \\ 3 Jefferson Lab, 12000 Jefferson Avenue, Newport News, VA 23606, USA \\ ${ }^{4}$ Department of Physics, Tokyo Woman's Christian University, Suginami-ku, Tokyo 167-8585, \\ Japan
}

\begin{abstract}
Nuclear parton distributions and structure functions are determined in an effective chiral quark theory. We also discuss an extension of our model to fragmentation functions.
\end{abstract}

Effective chiral quark theories are powerful tools to incorporate the nucleon quark substructure into the physics of nuclei. For example, the EMC effect[1] has shown that the quark distributions of bound nucleons differ from those of free nucleons, and this effect can be explained if one takes into account the response of the quark wave function to the mean fields inside the nucleus[2]. In this work, we concentrate on the model predictions for polarized nuclear structure functions, and also briefly discuss an extension to describe fragmentation functions[3].

We will be concerned with the following EMC ratios:

$$
R(x)=\frac{F_{2 A}\left(x_{A}\right)}{Z F_{2 p}(x)+N F_{2 n}(x)}, \quad R_{s}^{H}(x)=\frac{g_{1 A}^{H}\left(x_{A}\right)}{P_{p}^{H} g_{1 p}(x)+P_{n}^{H} g_{1 n}(x)} .
$$

Here $x$ is the usual Bjorken variable, and $x_{A}$ is $A$ times the Bjorken variable for the nucleus of mass number $A$. The structure functions of the nucleon (nucleus) are denoted as $F_{2}, g_{1}\left(F_{2 A}, g_{1 A}^{H}\right.$ with $H$ spin spin projection along the beam direction). $P_{\alpha}^{H}$ are the polarization factors of protons and neutrons. Both ratios in Eq.(1) become unity in a naive nonrelativistic single particle model.

Usually only a few valence nucleons (or holes) contribute to the nuclear polarization, and $g_{1 A}^{H}$ is of order $1 / A$ relative to $F_{2 A}$. Also, the structure function of a proton is larger and better known than that of the neutron. Therefore, possible candidates for the observation of the polarized EMC effect are stable nuclei which are not too heavy, and where the polarization is dominated by protons.

In our calculations, we describe the nucleon as a bound state of a quark and a diquark by using the Faddeev framework in the Nambu-Jona-Lasinio (NJL) 
model [4]. We take into account the scalar and axial vector diquark channels, and include confinement effects by eliminating unphysical quark decay thresholds in the proper time regularization scheme[5].

We calculate the unpolarized (polarized) quark distribution functions in the single nucleon by inserting the operator $\gamma^{+} \delta\left(x-k_{-} / p_{-}\right)\left(\gamma^{+} \gamma_{5} \delta\left(x-k_{-} / p_{-}\right)\right)$ into the quark propagators which make up the nucleon Green function. Finite nuclei in our present approach are described in a simple independent particle picture by using depth parameters of the scalar and vector potentials from our earlier self consistent nuclear matter calculations [5] and standard values for the range and diffuseness. Using the Faddeev equation we translate these potentials into the average fields for quarks, and use them in the calculation of the quark distributions in a bound nucleon. Finally, we calculate the momentum distributions of the nucleons[2], and obtain the quark distributions in the nucleus and the nuclear structure functions by using the convolution formalism.

The resulting EMC ratios are shown in Figs. 1-3 for the nuclei ${ }^{7} \mathrm{Li},{ }^{11} \mathrm{~B}$ and ${ }^{27} \mathrm{Al}$. It is seen that the polarized EMC effect is predicted to be larger than the unpolarized one. This corresponds to a reduction of the quark spin sum in the nucleus, i.e., some amount of the quark spin is converted into orbital angular momentum.

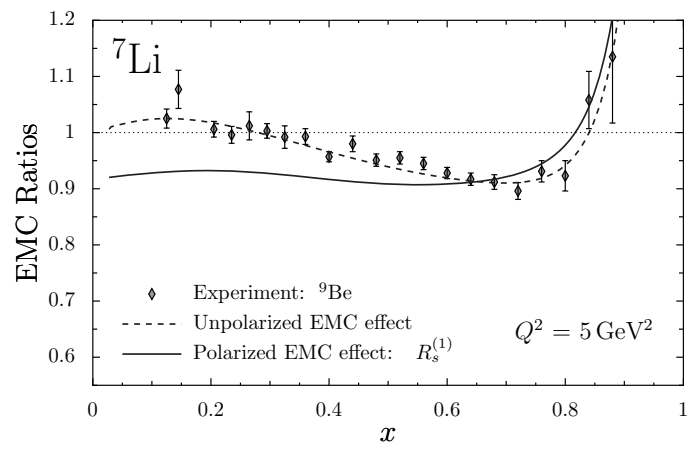

Figure 1. EMC ratios for ${ }^{7} \mathrm{Li}$. The experimental data refer to ${ }^{9} \mathrm{Be}$.

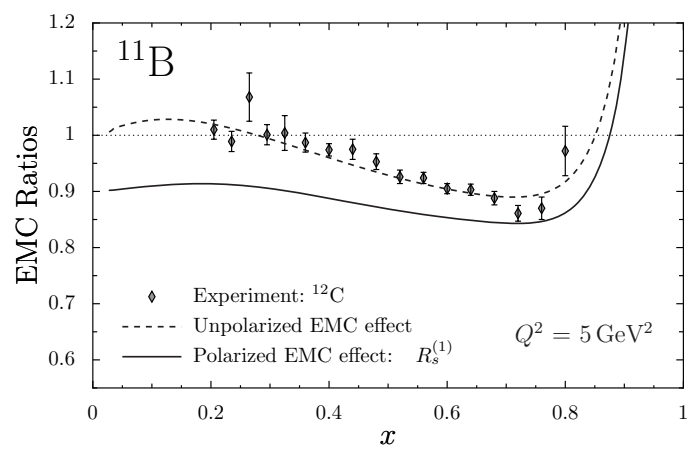

Figure 2. EMC ratios for ${ }^{11} \mathrm{~B}$. The experimental data refer to ${ }^{12} \mathrm{C}$.

Here we comment on a possible extension of the model to fragmentation 


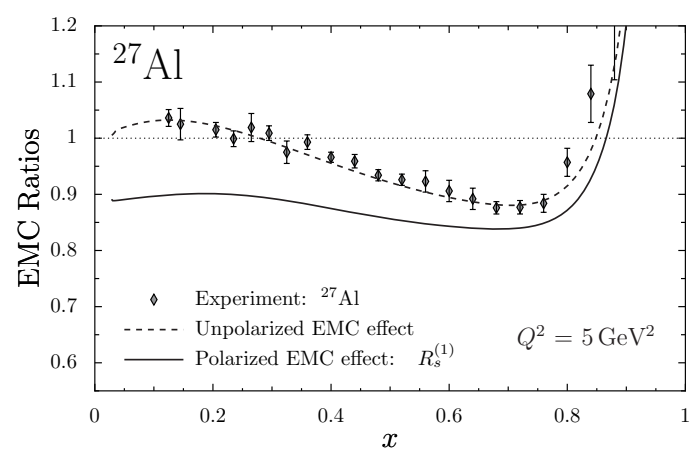

Figure 3. EMC ratios for ${ }^{27} \mathrm{Al}$.

functions: Starting from the operator definitions, one can use crossing and charge conjugation symmetries to show that the distribution $(f(x))$ and fragmentation $(D(z))$ functions are essentially one and the same function, defined in different regions of the variable. That is, if we define $f(x)=\Theta(1-x) F(x)$, then

$$
D(z)= \pm \Theta(1-z) \frac{z}{6} F\left(x=\frac{1}{z}\right),
$$

where the plus (minus) sign holds if the hadron is a fermion (boson). This relation is known in the literature as Drell-Levy-Yan (DLY) relation [6], and holds also in QCD up to the leading order. Numerical results and detailed discussions based on this relation will be presented in a future publication [3].

Acknowledgement. The $Q^{2}$ evolutions were performed by using the NLO computer codes of Ref.[7]. This work was supported by: Department of Energy, Office of Nuclear Physics, contract no. DE-AC02-06CH11357, under which UChicago Argonne, LLC, operates Argonne National Laboratory; contract no. DE-AC05-84ER40150, under which JSA operates Jefferson Lab, and by the Grant in Aid for Scientific Research of the Japanese Ministry of Education, Culture, Sports, Science and Technology, project no. C-19540306.

\section{References}

1. M. Arneodo, Phys. Rep. 240 (1994) 301.

2. I.C. Cloët, W. Bentz, and A.W. Thomas, Phys. Lett. B 642 (2006) 210.

3. W. Bentz, T. Ito, A.W. Thomas, and K. Yazaki, to be published.

4. N. Ishii, W. Bentz, and K. Yazaki, Nucl. Phys. A587 (1995) 617.

5. W. Bentz and A.W. Thomas, Nucl. Phys. A 696 (2001) 138.

6. S.D. Drell, D.J. Levy, and T.M. Yan, Phys. Rev. 187 (1969) 2159; Phys. Rev. D 1 (1970) 1617.

7. M. Miyama and S. Kumano, Comput. Phys. Commun. 94 (1996) 185;

M. Hirai, S. Kumano, and M. Miyama, Comput. Phys. Commun. 108 (1998) 38 . 